\title{
A quantum Interferometer for quantum gravity studies
}

Losero, E.; Tekuru, S. Pradyumna; Traina, P.; Ruo-Berchera, I.; Zucco, M.; Degiovanni, I. P.; Jacobsen, C. S.; Gehring, Tobias; Andersen, Ulrik Lund; Genovese, M.

Published in:

Quantum Communications and Quantum Imaging XVII

Link to article, DOI:

$10.1117 / 12.2527471$

Publication date:

2019

Document Version

Publisher's PDF, also known as Version of record

Link back to DTU Orbit

Citation (APA):

Losero, E., Tekuru, S. P., Traina, P., Ruo-Berchera, I., Zucco, M., Degiovanni, I. P., Jacobsen, C. S., Gehring, T., Andersen, U. L., \& Genovese, M.' (2019). A quantum Interferometer for quantum gravity studies. In K. S. Deacon (Ed.), Quantum Communications and Quantum Imaging XVII (Vol. 11134). [111340F] SPIE International Society for Optical Engineering. https://doi.org/10.1117/12.2527471

\section{General rights}

Copyright and moral rights for the publications made accessible in the public portal are retained by the authors and/or other copyright owners and it is a condition of accessing publications that users recognise and abide by the legal requirements associated with these rights.

- Users may download and print one copy of any publication from the public portal for the purpose of private study or research.

- You may not further distribute the material or use it for any profit-making activity or commercial gain

- You may freely distribute the URL identifying the publication in the public portal 


\section{A quantum Interferometer for quantum gravity studies}

E. Losero, S. Pradyumna Tekuru, P. Traina, I. RuoBerchera, M. Zucco, et al.

E. Losero, S. Pradyumna Tekuru, P. Traina, I. Ruo-Berchera, M. Zucco, I. P. Degiovanni, C. S. Jacobsen, T. Gehring, U. L. Andersen, M. Genovese, "A quantum Interferometer for quantum gravity studies," Proc. SPIE 11134, Quantum Communications and Quantum Imaging XVII, 111340F (6 September 2019); doi: 10.1117/12.2527471

SPIE Event: SPIE Optical Engineering + Applications, 2019, San Diego, California, United States 


\title{
A quantum Interferometer for quantum gravity studies
}

\author{
E. Losero ${ }^{a, b}$, S. Pradyumna Tekuru ${ }^{a, b}$, P. Traina ${ }^{a}$, I. Ruo-Berchera ${ }^{a}$, M. Zucco $^{a}$, I. \\ P. Degiovanni ${ }^{a}$, C. S. Jacobsen ${ }^{d}$, T. Gehring ${ }^{d}$, U. L. Andersen ${ }^{d}$, M. Genovese $^{a, c}$ \\ ${ }^{a}$ INRIM, Strada delle Cacce 91, 10135, Torino, Italy \\ ${ }^{b}$ DET, Politecnico di Torino, Corso Duca degli Abruzzi 24, 10129, Torino, Italy \\ ${ }^{c}$ INFN, sezione di Torino, via P. Giuria 1, 10125 Torino, Italy \\ ${ }^{d}$ Center for Macroscopic Quantum States (bigQ), Department of Physics, Technical University of \\ Denmark, Fysikvej, Denmark
}

\section{ABSTRACT}

The search for Planck scale effects is one of holy grains of physics. At Fermilab, a system of two Michelson interferometers (MIs) was built for this purpose: the holometer. This device operates using classical light, and, therefore, its sensitivity is shot-noise limited. In collaboration with the Danish Technical University, we built a proof of principle experiment devoted to experimentally demonstrate how quantum light could improve the holometer sensitivity below the shot noise limit. It is the first time that quantum light is used in a correlated interferometric system. In particular the injection of two single mode squeezed state (one in each interferometer) and of a twin-beam state is considered, and the system performance compared in the two cases. In this proceeding, after a general introduction to the holometer purposes and to our experimental set-up, we present some characterization measurements concerning the quantum light injection.

\section{INTRODUCTION}

Optical interferometry represents one of the best sensing tool for detection of faint phase noises. A prime example is the recent detection of gravitational waves [1]. Since, the interferometer sensitivity is constrained by the shot noise, one could increase the power in order to enhance the sensitivity. However, further increasing of power would lead to increase of radiation pressure noise from the mirrors, thermal effects, scattering etc. In order to mitigate this problem, a sub-shot-noise phase measurement in a MI by injecting squeezed state was suggested [2] and has already been realized in many gravitational wave detectors [3].

There are fundamental sources of noises such as holographic noise [4] or gravitational wave background $[5 ; 6]$ that could produce correlated phase fluctuations in two separated interferometers: detect this correlation considering the correlation of the two MIs output is the aim of a holometerlike system. In particular, holographic noise would arise due to non-commutative property of the fundamental geometrical variables conjectured in some Planck scale phenomenological models $[7 ; 8]$. It has been argued that a MI allows to "accumulate" this noise, leading to potentially

Further author information: M. Genovese: E-mail: m.genovese@inrim.it 
measurable effects. If the two interferometers occupy the same space-time volume this noise is expected to induce correlated phase fluctuations in the two MIs. Moreover, this noise should be present in the frequencies up to $\mathrm{MHz}$ range. For this purpose, a double interferometer system, named holometer, consisting of pair of $40 \mathrm{~m}$ long power recycled MIs placed close to each other, has been built at the Fermilab $[9 ; 10]$. At this moment, the holometer is being operated using classical light only. The results of the first measurements were reported in [10].

We build a first proof-of-principle experiment for demonstrating the possibility of overcoming the classical limits in correlated phase detection sensitivity by exploiting the properties of quantum states of light in a double interferometric system, analog to the Fermilab holometer but at smaller scale (arm length around $1 \mathrm{~m}$ ). This has been motivated by the results of earlier theoretical works $[11 ; 12]$. A brief theoretical description of the scheme as well as a brief description of the experimental setup is presented. Moreover, different measurements characterizing the quantum light injected are discussed.

\section{THEORETICAL DESCRIPTION}

The general idea is represented in Fig. 1. Aim of the measurement is to measure the covariance between the two interferometer phases, $\operatorname{Cov}\left(\phi_{1}, \phi_{2}\right)$.

Let us consider two MIs placed closed to each other and fed with two coherent states $\left|\mu e^{i \psi}\right\rangle$ from the $b_{k}$ input ports, $k=1,2$. A quantum state $|\Psi\rangle$ is injected from the anti-symmetric ports $a_{k}$, classically unused. The observable $\hat{C}\left(\phi_{1}, \phi_{2}\right)$ between the outputs of the interferometer is measured. From $\hat{C}\left(\phi_{1}, \phi_{2}\right)$ it will be then possible to retrieve the correlation in the phases fluctuation. The observable $\hat{C}$ should be chosen accordingly to the input quantum states considered, in order to minimize the uncertainty on $\operatorname{Cov}\left(\phi_{1}, \phi_{2}\right)$.

Two types of quantum states $|\Psi\rangle$ were considered to be injected into each interferometer:

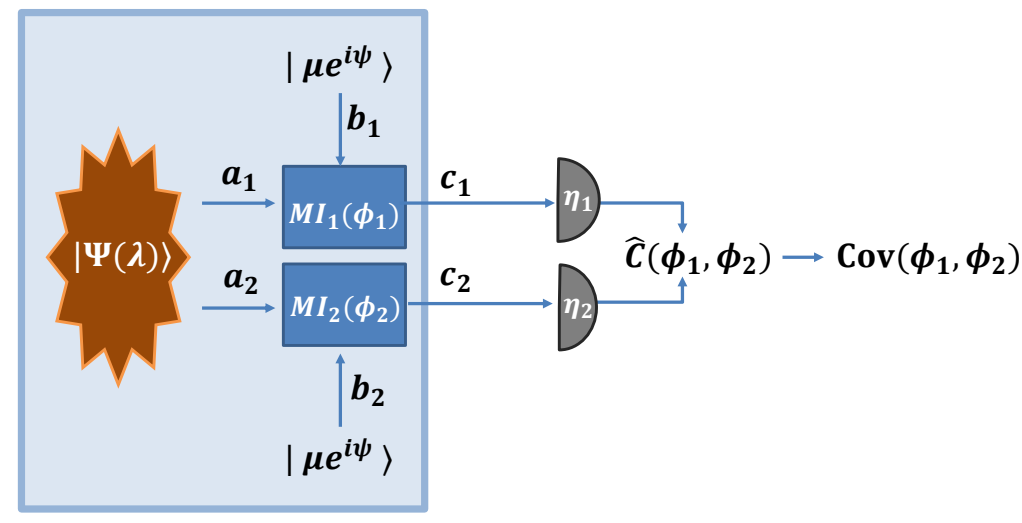

Figure 1: Scheme representing the general idea of a quantum enhanced correlation measurement using a couple of interferometers. $I_{1}\left(\phi_{1}\right)$ and $I_{2}\left(\phi_{2}\right)$ are the two Michelson interferometers (MIs) and they are fed by the coherent state $\left|\mu e^{i \psi}\right\rangle$. A quantum bipartite state $|\Psi(\lambda)\rangle$ can be sent in the interferometers from the classically unused port. From measuring the observable $\hat{C}\left(\phi_{1}, \phi_{2}\right)$ the correlation of the two interferometers phases $\phi_{1}$ and $\phi_{2}$ can be estimated. The use of quantum light can enhance the sensitivity of the measurement respect to the situation where only the coherent states are used. 
- Two independent squeezed light states: $|\Psi\rangle=|s q z\rangle_{1} \otimes|s q z\rangle_{2}$.

When independent squeezed vacuum states are injected into the interferometer, $\hat{C}\left(\phi_{1}, \phi_{2}\right)$ is chosen as the product of the photon number operators detected at the output port: $\hat{C}=$ $\hat{N}_{1} \hat{N}_{2}$. However, to remove the DC component, we evaluate

$$
\hat{C}\left(\phi_{1}, \phi_{2}\right)=\left(\hat{N}_{1}-\left\langle\hat{N}_{1}\right\rangle\right)\left(\hat{N}_{2}-\left\langle\hat{N}_{2}\right\rangle\right)
$$

It can be demonstrated that the uncertainty on the covariance, while working close to the dark fringe $\left(\phi_{1}=\phi_{2} \approx 0\right)$, is given by

$$
\mathscr{U}^{(0)}\left(\operatorname{Cov}\left(\phi_{1}, \phi_{2}\right)\right)_{s q z}=\sqrt{2 \frac{e^{-2 r_{1}}}{\mu}+\frac{\left(1-\eta_{1}\right)}{\eta_{1} \mu}} \sqrt{\frac{e^{-2 r_{2}}}{\mu}+\frac{\left(1-\eta_{2}\right)}{\eta_{2} \mu}}
$$

where $\eta_{1}$ and $\eta_{2}$ are the detector efficiencies in the two interferometers, $r_{1}$ and $r_{2}$ are the two squeezing factors and $\mu$ is the number of photons in the coherent beam. In the ideal case of negligible losses, i.e. for $\eta_{1}=\eta_{2}=1$, the uncertainty in the covariance of the phase is given by $\mathscr{U}^{(0)}\left(\operatorname{Cov}\left(\phi_{1}, \phi_{2}\right)\right)_{s q z}=\frac{\sqrt{2} e^{-r_{1}} e^{-r_{2}}}{\mu}$.

- Two mode squeezed states $|\Psi\rangle=|t w b\rangle_{1,2}$.

When each mode of a two mode squeezed state, also named twin-beam state, is injected into the interferometers, we chose $\hat{C}\left(\phi_{1}, \phi_{2}\right)=\left(\hat{N}_{1}-\hat{N}_{2}\right)^{2}$. The results of the theoretical uncertainty reduction in correlated phase detection are thoroughly discussed in [11; 12].

It emerges that $\mathscr{R}^{(0)}=\frac{\mathscr{U}_{t w b}^{(0)}}{\mathscr{U}_{c l}^{(0)}}$ can be divided into two regimes respect to $\phi$ : photon number entanglement regime and quadrature correlations regime. The first regime corresponds to extremely high efficiencies and $\phi$ very close to the dark fringe: in this case the classical field component is almost zero, $(1-T) \mu \ll T \lambda$ (where $T=\cos ^{2}(\phi / 2)$ is the transmission factor at the MI outputs, $\mu$ is the mean photon number in the coherent field and $\lambda$ is the mean photon number in the twin-beam state). The photon number correlation from the injected twin beam state dominates, giving a dramatic reduction in the uncertainty on $\operatorname{Cov}\left(\phi_{1}, \phi_{2}\right)$. In principle $\mathscr{U}^{(0)}=0$ can be obtained. In the second regime, for $T \lambda \ll(1-T) \mu$ and slightly away from dark fringe, quadrature correlations dominate and hence the uncertainty reduction behaves as same as for the case of independent squeezed states. Experimentally, this second regime can offer interesting advantages while working in the first regime is too demanding for current technologies (in particular $\eta$ extremely close to 1 would be necessary).

\section{EXPERIMENTAL SETUP - CLASSICAL PART}

Our system consists of two power recycling MIs placed close to each other with small separation between the two beam-splitters. Fig. 2 depicts the simplified schematic of the experimental setup, concerning the classical part. The quantum light production and injection is presented in the next section. A Nd-YAG laser with a maximum output power of $300 \mathrm{~mW}$ at wavelength $1064 \mathrm{~nm}$ is used. Around $1.5 \mathrm{~mW}$ are injected into each interferometer, part of the light is also used for generation of the squeezed light. For having an efficient $\mathrm{TEM}_{00}$ input mode, the light from the 


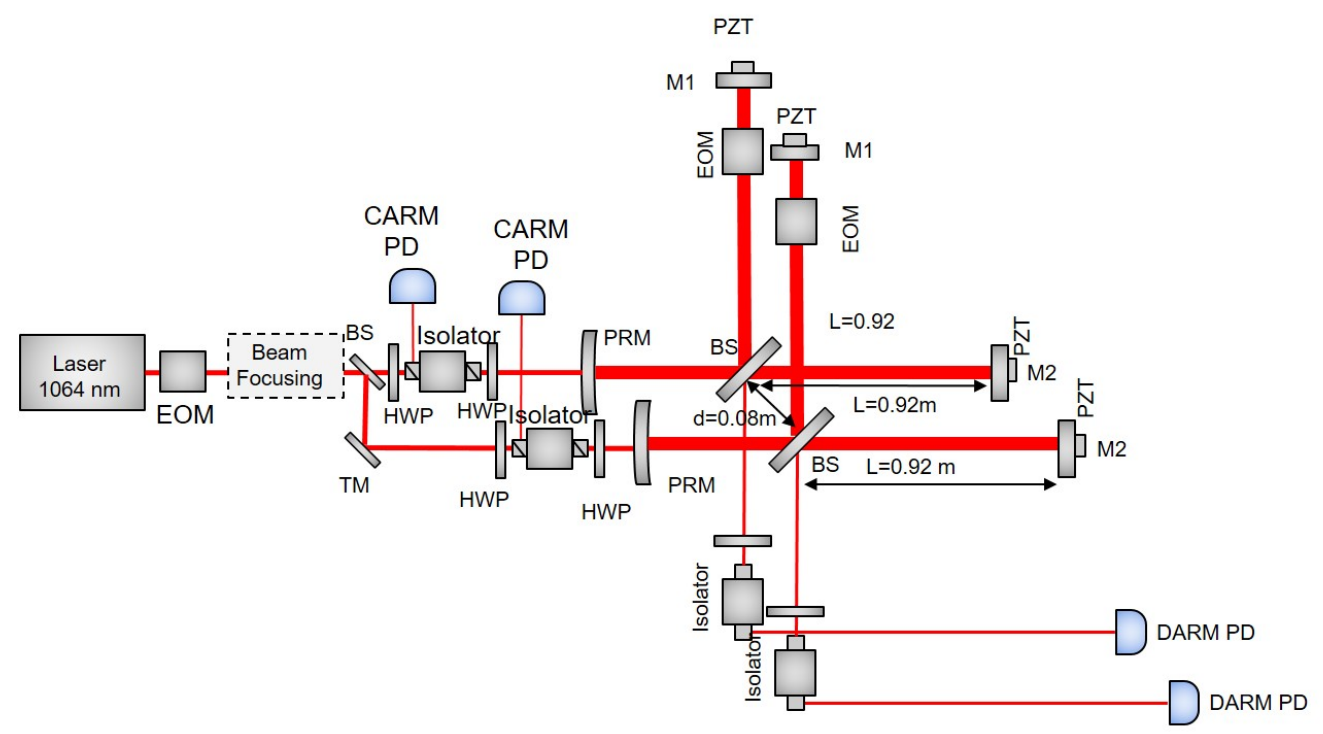

Figure 2: Simplified schematic of the double-interferometer setup, concerning the classical part. Two MIs with arm length $L=0.92 \mathrm{~m}$ were co-located, with a distance between the two beam splitters (BSs) of around $8 \mathrm{~cm}$. M1, M2: piezo-actuated high-reflectivity (99.9\%) end mirrors. PRM: partially reflecting (90\%) power recycling mirror, radius of curvature $r_{c}=1.5 \mathrm{~m}$.; HWP: Half waveplate; PD: Photo-detector; BS: balanced beam splitter.

laser is fiber coupled before injection into the interferometers. Each interferometer is formed by a power recycling mirror $(P R M)$ having radius of curvature of $1.5 \mathrm{~m}$, end plain mirrors $M 1$ and M2 (reflectivity around 99.9\%) stabilized by a PZT actuators attached and a 50-50 beam splitter. The length of each arm of the interferometer is around $92 \mathrm{~cm}$. The distance between the two beam splitters was around $8 \mathrm{~cm}$. A set of lenses are placed before the interferometers for mode matching the power recycling interferometer. An InGaAs photo-diode with high quantum-efficiency (99\%) and low noise (Noise Equivalent Power $1.210^{-11} \mathrm{~W} / \sqrt{\mathrm{Hz}}$ ) is used to detect the output of the system.

It is necessary to lock the system to the proper working point, in order to get rid of environmental noise and reach the desired sensitivity. In particular we work close to the dark fringe (output power $\sim 0$ ) and at the cavity resonance. The first condition implies for the differential arm length, DARM: $L_{1}-L_{2}=n \lambda+\delta$ while the second condition implies for the common arm length: CARM $L_{e f f}=\frac{L_{1}+L_{2}}{2}=m \lambda$, being $L_{1}$ and $L_{2}$ the length of the two arms. The details of the locking scheme and squeezed light source generation are thoroughly discussed in [13].

\section{SQUEEZING GENERATION AND CHARACTERIZATION MEASUREMENTS}

The squeezed light source is schematically depicted in Fig. 3. It is based on parametric downconversion process in a periodically poled potassium titanyl phosphate crystal (ppKTP) inside a cavity. A semi-monolithic linear cavity, constituted by the edges of the crystal and a semitransparent mirror of reflectivity $90 \%$, is used. For locking the cavity length to the resonance, the cavity is seeded with $1064 \mathrm{~nm}$ light, opportunely phase modulated at $37.22 \mathrm{MHz}$ (or $36.5 \mathrm{MHz}$ ) by 


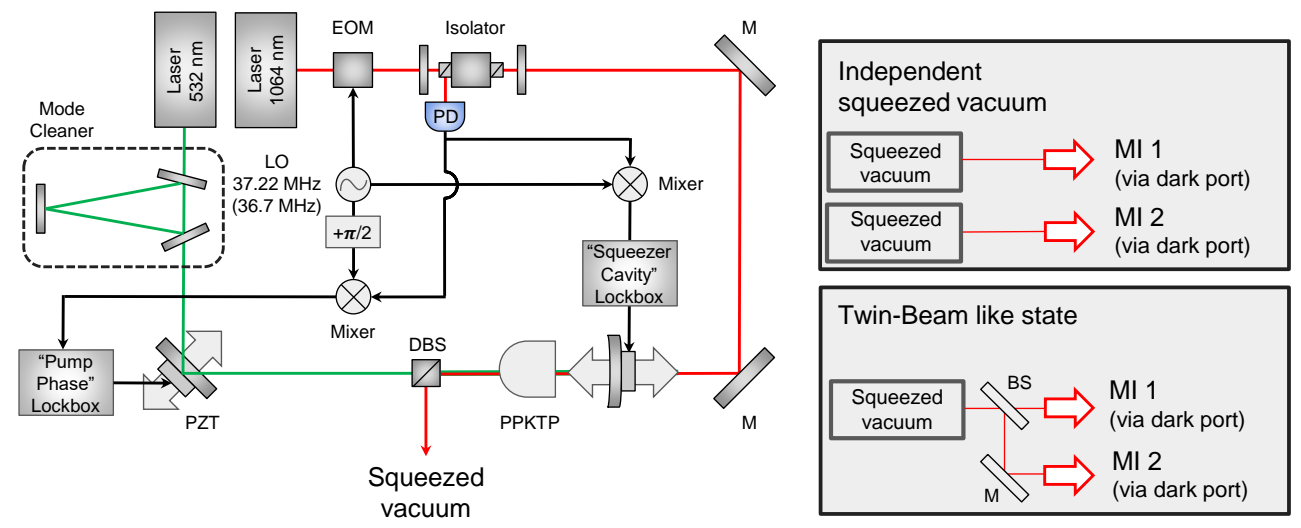

Figure 3: Experimental set-up of each squeezer. PPKTP: potassium titanyl phosphate crystal; DBS: dichroic beam splitter; PZT: piezoelectric actuators; EOM: electro-optical modulator; LO: local oscillator; PD: photo-diode

a EOM. We refer to this beam as the control beam. The PDH technique is used [14] and the error signal is sent to the PZT actuator attached to the cavity mirror.

The same laser source used for producing the control beam also provides $532 \mathrm{~nm}$ radiation by an internal module for second harmonic generation. We refer to this beam as pump beam. In order to have a clean $\mathrm{TEM}_{00}$ mode the pump beam is sent trough a mode-cleaner. After that it is directed to the locked cavity, where the parametric down conversion process takes place. To establish a phasematch condition between the pump and the control beam, the crystals temperature is actively stabilised. According to [15], the phase relation between pump and control beam determines the orientation of the squeezing ellipse and therefore needs to be locked appropriately. In particular, if we lock to the bright fringe i.e to the amplification, the squeezing results in the phase quadrature while if we lock it to de-amplification it results in amplitude quadrature. The error signal for locking to the amplitude squeezing is sent to a PZT actuator mounted on a mirror on the pump path. Finally, a dichroic beam splitter (DBS) is used at the output of the squeezed source to separate the $1064 \mathrm{~nm}$ component from the (undesired) $532 \mathrm{~nm}$ one. Summarizing, the output of the squeezer consists in a displaced squeezed state with displacement provided by the control beam. The squeezed state generated is very sensitive to losses, therefore high reflective mirrors $(R \sim 99 \%)$ need to be used.

Note that, in a preliminary phase, the control beam can be extremely useful for alignment and mode-matching optimization since it perfectly spatially overlaps the generated squeezed light.

\subsection{Characterisation measurements for the quantum states}

To detect the squeezing level of the light produced by the squeezer described above, a homodyne measurement is performed. A scheme of this measurement is reported in Fig. 4: the squeezed beam and the local oscillator are interfered on a BS. The phase is periodically varied by a phaseshifter and the subtracted output is sent to a spectrum analyzer. This kind of measurement allows to access the quadrature of the field, thus allowing to detect the noise reduction corresponding to squeezing injection. The output of the spectrum analyzer, working at $13.5 \mathrm{MHz}$ in the zero-span mode, is presented in Fig. 5. The blue line refers to the coherent case, where the squeezed beam is 


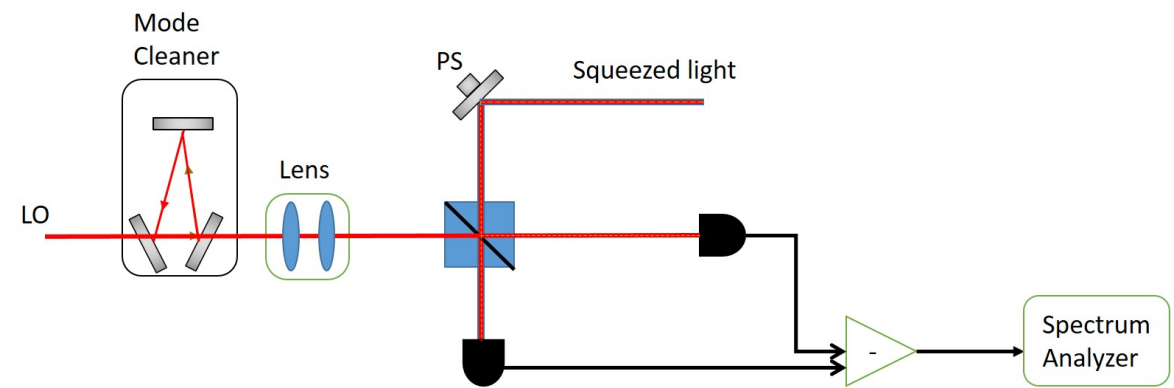

Figure 4: Scheme of the homodyne measurement, which allows to have experimental access to the field quadratures. The squeezed beam and local oscillator are interfered. The phase is varied by a phase shifter and the subtracted output is sent to a spectrum analyzer.

blocked and the measurement is performed with only the LO, at the considered frequency we are shot-noise limited. The red curve refers to the squeezed case; both curves are normalized to the coherent case. As we modulate the phase between the local oscillator and the squeezed light, the

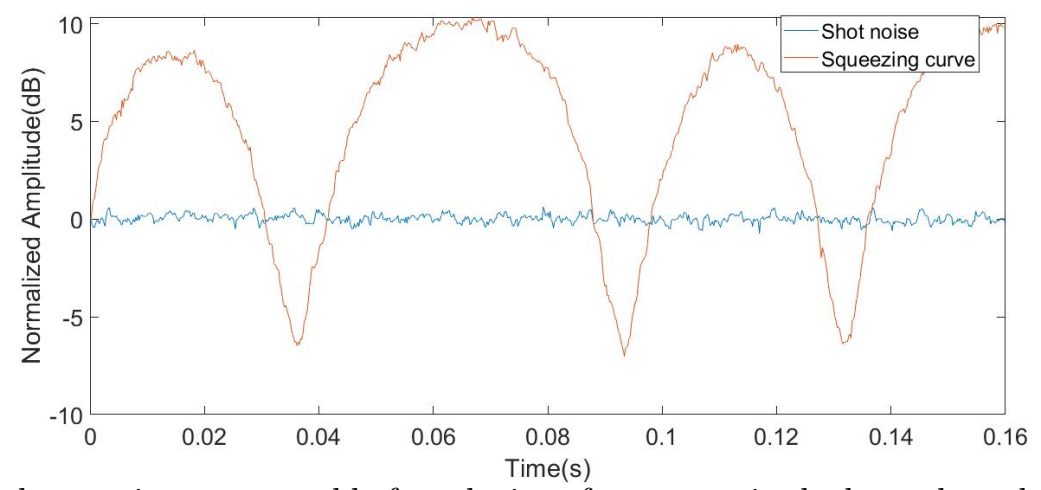

Figure 5: Initial squeezing, measured before the interferometer via the homodyne detection scheme in function of time. At varying the time the phase shift between LO and the squeezed field is periodically modulated. The blue line represents the shot noise level while the red line corresponds to the squeezing injection.

variance varies from anti-squeezing quadrature $V_{a n t i-s q}$ to squeezing quadrature $V_{s q}$. In our case we measured:

$$
V_{s q}=6.5 d B, \quad V_{\text {anti-sq }}=10 d B
$$

From this values it is possible to estimate the squeezing level at the source, and the efficiency of the system. In particular, the following equations hold:

$$
\begin{gathered}
V_{s q}=\eta V+(1-\eta) \\
V_{\text {anti-sq }}=\eta / V+(1-\eta)
\end{gathered}
$$

where $V=e^{-2 r}$ is the amount of squeezing produced by the squeezer, and $\eta=\eta_{t} \cdot \eta_{m}$ includes both the transmission efficiency from the squeezer cavity to the photo-detector $\left(\eta_{t}\right)$ and the modematching between the squeezed beam and the LO $\left(\eta_{m}\right)$. Solving Eq. 5.1.2-5.1.3 respect to $V$ and 
$\eta$, and having independently estimated $\eta_{m}=0.99$, the following values are obtained:

$$
V=10.6 d B, \quad \eta_{t}=0.85
$$

We conclude that our source produce a squeezed state of around $10.6 \mathrm{~dB}$, however, losses induced by optical components and mode-matching will necessarily decrease the squeezing level inside the interferometers.

We report here the measurements for evaluating the squeezing level inside the interferometers, when two independent squeezed states are injected $\left(|\Psi\rangle=|s q z\rangle_{1} \otimes|s q z\rangle_{2}\right)$.

In Fig. 6i the photon noise normalized to the shot noise level in function of time time is reported. For performing this measurement the interferometer is locked at the typical working point and the phase shifter on the squeezed beam path is modulated. The blue curve is the shot noise level, while the green curve is the noise in case of squeezing injection.

The phase is then locked to the squeezed quadrature, in order to reduce the noise below the shot noise level. Fig. 6ii shows the photon noise, normalized to the shot noise level, in a frequency bandwidth around $13.5 \mathrm{MHz}$, for the two interferometers. The red line and the blue line correspond to the coherent case and the squeezed states injection respectively. The achieved squeezing level is of $2.4 \mathrm{~dB}$ for the first $\mathrm{MI}$ and $3 \mathrm{~dB}$ for the second one.

For experimentally reproduce the measurement strategy with $|\Psi\rangle=|t w b\rangle_{1,2}$ we use an approximate twin-beam state. In particular this state is obtained splitting at a balanced beam splitter one single-mode squeezed beam (See Fig. 3, panel on the right). The resulting bipartite state does not present photon-number entanglement between the two modes, but, considering one of the two quadrature, it present exactly the same quadrature correlation as a real twin-beam. Since the level of losses present in our system necessarily imply to work in the quadrature correlation regime, the approximate state will behave exactly as an actual twin beam state. Each mode of the twin beam like state is injected in the two interferometer and subtraction between the outputs is measured: the non-classical quadrature correlation is expected to provide a noise reduction in the output subtraction. When the twin-beam like state is injected in the interferometers, we expect a non zero covariance between the two outputs. The cross correlation between the two outputs is evaluated both in coherent case and in twin beam case, as shown in Fig. 7. For the twin beam case (panel on the right) there is a non-zero correlation at delay $\tau=0$, while it vanishes in the classical case (panel on the left). 


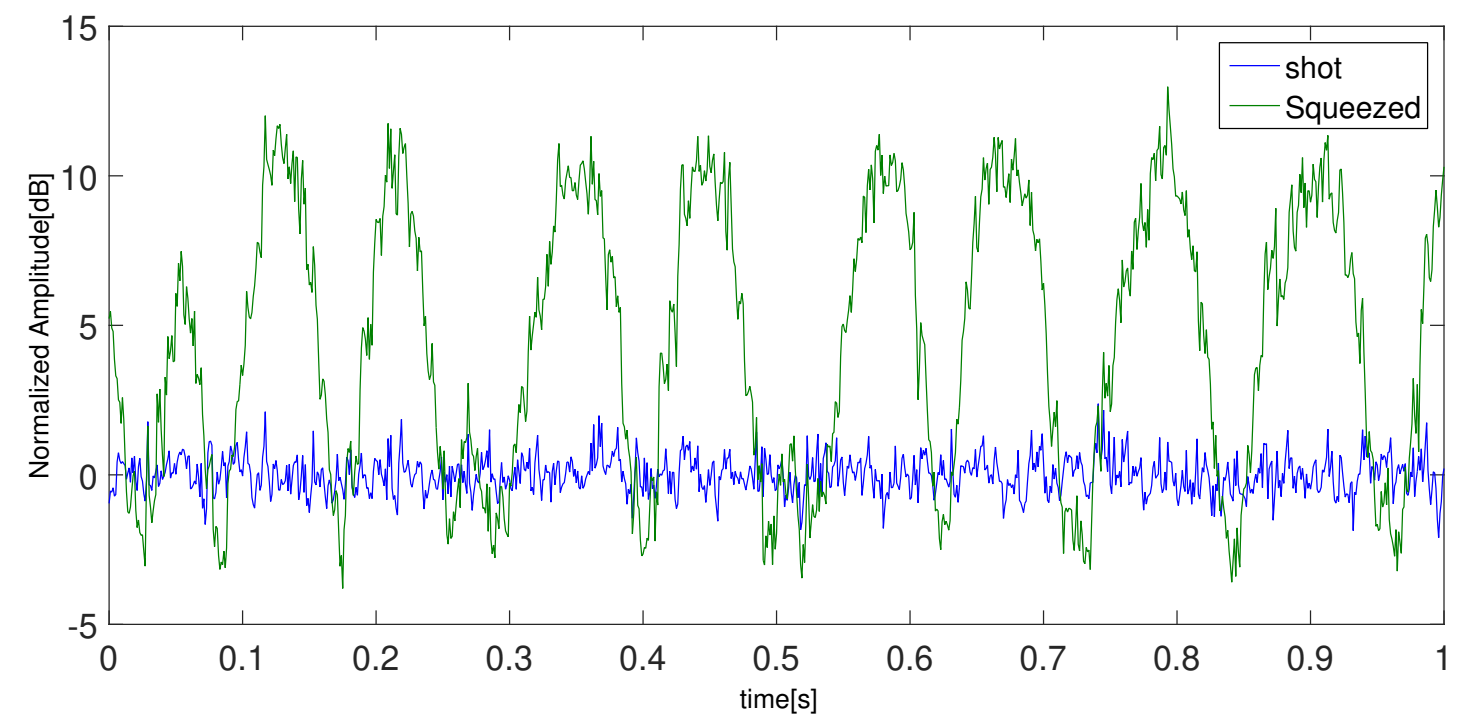

(i)
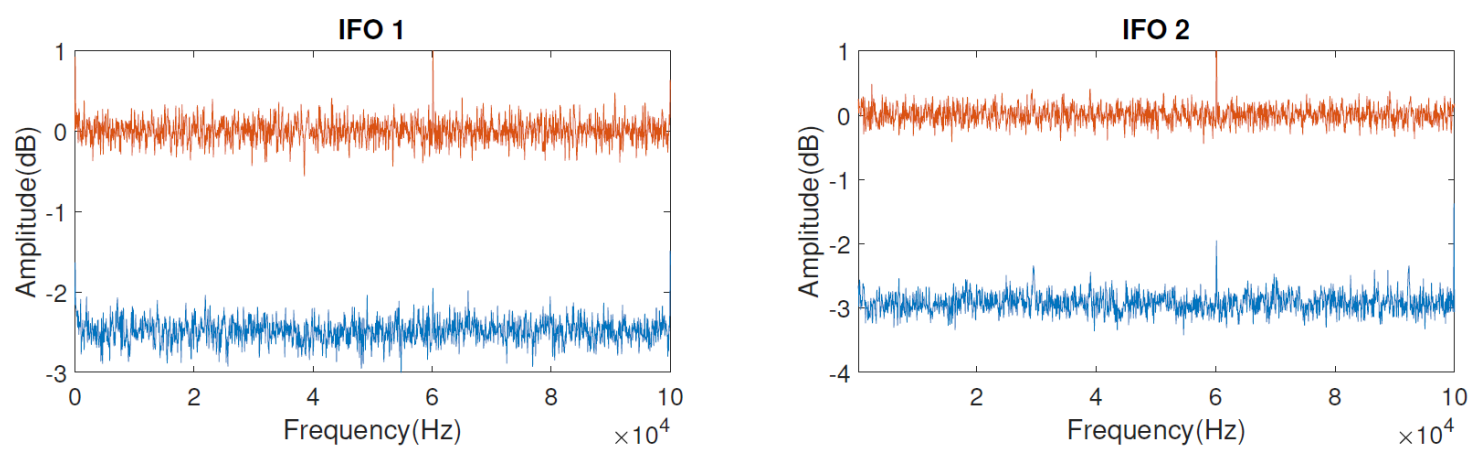

(ii)

Figure 6: (i) Normalized shot noise as a function of phase between the squeezer and the interferometer recorded on a spectrum analyzer. The blue curve represents the shot noise level and the green curve represents the photon noise in correspondence of squeezing injection. (ii) Photon noise with (blue curve) and without (red curve) squeezing injection, after locking to the squeezed quadrature. Squeezing injection allows to reduce the noise below the shot noise level. 

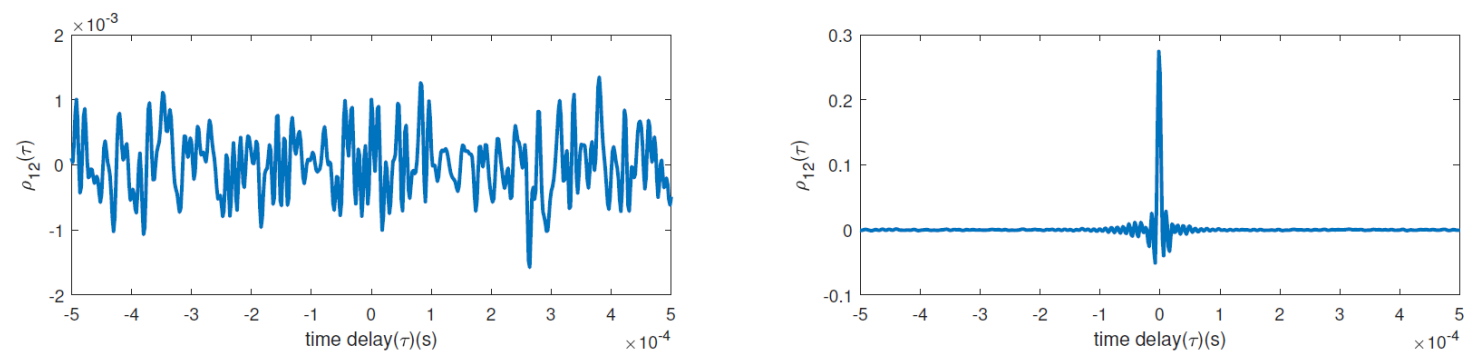

Figure 7: Cross-correlation coefficient between the two interferometers output as a function of time delay $\tau$. The figure on the left corresponds to the classical case and the figure on the right corresponds to the twin-beam like state injection. In this case there is a non-zero covariance at $\tau=0$. 


\section{CONCLUSION}

We presented some characterization measurements for the quantum states injected into our holometerlike system. In particular, we focused on two possibilities: two single mode squeezed states and a twin beam like state. We measured $6.5 \mathrm{~dB}$ of squeezing at the output of the squeezer. Losses inside the interferometer lower the squeezing level in the two MIs. In particular a squeezing level of 2.4 $\mathrm{dB}$ and $3 \mathrm{~dB}$ respectively is demonstrated. The twin beam like state is obtained splitting a single mode squeezed state into two beams using a balanced beam splitter. The presence of correlations between these two beams has been measured, as well as a remaining squeezing level of $0.8 \mathrm{~dB}$ and $1 \mathrm{~dB}$. The quadrature correlations between the two modes lead to a collective enhancement of 2 $\mathrm{dB}$ in the output subtraction.

Using these state of light it is possible to enhance the sensitivity of the system, allowing to detect correlations in the phase fluctuation below the shot-noise limit, classically unavoidable. This result has possible applications in fundamental research, such as detection of holographic noise, but also detection of gravitational wave background or search for traces of primordial black-holes $[16 ; 17]$.

\section{References}

[1] B. P. Abbott et al. Observation of gravitational waves from a binary black hole merger. Physical Review Letters, 116(6):061102, 2016.

[2] Carlton Caves. Quantum-mechanical noise in an interferometer. Physical Review D, 523(8):1693-1708, 1981.

[3] J. Aasi et al. Enhanced sensitivity of the LIGO gravitational wave detector by using squeezed states of light. Nature Photonics, 7(8):613-619, 2013.

[4] Craig J Hogan. Interferometers as probes of Planck in a quantum geometry. Physical Review D, 85(6):064007, 2012.

[5] Tomotada Akutsu et al. Search for a stochastic background of 100-MHz gravitational waves with laser interferometers. Physical Review Letters, 101(10):101101, 2008.

[6] Joseph D. Romano and Neil J. Cornish. Detection methods for stochastic gravitational-wave backgrounds: A unified treatment. Living Reviews in Relativity, 20(2):1-223, 2017.

[7] Craig J. Hogan, O. Kwon. Models of exotic interferometer cross-correlations in emergent space-time. Class and Q.Grav, 35(20):204001, 2018.

[8] Craig J. Hogan, O. Kwon. Statistical models of exotic rotioanal correlations in emergent space-time. Class and Q.Grav, 34(13):135006, 2017.

[9] Aaron S.Chou et al. MHz gravitational wave constraints with Decameter Michelson interferometers. Physical Review D, 95(6):063002, 2017.

[10] Aaron S.Chou et al. First Measurements of High Frequency Cross Spectra from a pair of Large Michelson Interferometers. Physical Review letters, 117(11):1111102, 2016.

Proc. of SPIE Vol. 11134 111340F-10 
[11] Ivano Ruo berchera, I.P. Degiovanni, Stefano Olivares, N. Samantaray, P.Traina and Marco Genovese. One and two mode squeezed light in correlated interferometery. Physical Review A, 92(5):053821, 2015.

[12] Ivano Ruo-Berchera, I.P. Degiovanni, Stefano Olivares and Marco Genovese. Quantum light in coupled interferometers for quantum gravity tests. Physical Review letters, 110(21):213601, 2013.

[13] S. Tekuru. An application of quantum metrology: enhanced correlated interferometry. Doctoral thesis Polito, 2019.

[14] Eric D. Black. An introduction to PoundDreverHall laser frequency stabilization. American Journal of Physics, 69, 79, 2001.

[15] S. Schiller G. Breitenbach and J. Mlynek. Measurement of the quantum states of squeezed light. Nature, 387, 471:475, 1997.

[16] B. J. Carr and S. W. Hawking. Black Holes in the early universe. Mon. Not. R. astr. Soc., 168:399-415, 1974.

[17] Aaron S. Chou et al. Interferometric constraints on quantum geometrical shear noise correlations. Classical and Quantum Gravity, 34:165005, 2017. 